Check for updates

Cite this: Phys. Chem. Chem. Phys., 2017, 19, 16477

Received 15th February 2017 Accepted 30th May 2017

DOI: $10.1039 / \mathrm{c7cp01011g}$

rsc.li/pccp

\title{
Enhanced growth of tellurium nanowires under conditions of macromolecular crowding
}

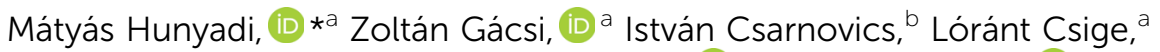 \\ Attila Csik, ${ }^{a}$ Lajos Daróczi, ${ }^{c}$ Róbert Huszánk (D) ${ }^{a}$ and Zoltán Szűcs (D) ${ }^{a}$
}

\begin{abstract}
An unprecedented rate enhancement was observed in the wet-chemical synthesis of tellurium nanowires under crowded conditions of inert macromolecules. The synthesis was carried out at $105{ }^{\circ} \mathrm{C}$ using solutions of sodium tellurite $\left(\mathrm{Na}_{2} \mathrm{TeO}_{3}\right)$ as a precursor, hydrazine $\left(\mathrm{N}_{2} \mathrm{H}_{4}\right)$ as a reducing agent, and polyvinylpyrrolidone (PVP) as both a stabilizing and crowding agent. The PVP concentration was systematically varied between the dilute and crowding regimes up to $166 \mathrm{~g} \mathrm{l}^{-1}$. The growth of the nanowires was monitored by measuring their size-dependent optical properties in the UV-Vis spectrum characterizing the size and morphology evolution of the nanowires and a coexisting phase of amorphous tellurium nanoparticles. The observed growth characteristics were interpreted in terms of non-specific structural organization of the crowded media due to the entropic-driven effects of space compartmentalization.
\end{abstract}

\section{Introduction}

It has long been recognized that macromolecular crowding (MMC) is an inevitable factor for the normal operation of life, which governs the chemistry of large organic molecules in physiological processes. ${ }^{1-6}$ In contrast, remarkably little attention has been paid to investigating crowding effects on the reaction kinetics or phase behaviour during the formation and stabilization of inorganic nanomaterials and how they can exert their designed functionality. ${ }^{7-10}$ In future medical applications, artificial and composite nanostructures entering living cells experience such crowded media of a variety of macromolecules as well as severe spatial restrictions. We might also ask whether the MMC acts on the underlying mechanism of such processes and potentially increases the thermodynamic activities of the building-block particles as a specific catalyst in the case of absorbing macromolecules or as a non-specific crowder through entropic effects of space exclusion and compartmentalization. ${ }^{11,12}$

Nanoparticles (NPs) are routinely synthesized in dilute systems utilizing phase transition in supersaturated solutions of lowmolecular weight species as their monomeric building blocks. In practice, the challenging task is to find an appropriate model system for NP synthesis to study crowding effects in the growth

\footnotetext{
${ }^{a}$ Institute for Nuclear Research, Hungarian Academy of Sciences, Bem tér 18/c, 4026 Debrecen, Hungary. E-mail: hunyadi.matyas@atomki.mta.hu; Fax: +36 52416 181; Tel: +36 52509270

${ }^{b}$ Department of Experimental Physics, Debrecen University, Bem tér 18/a, 4026 Debrecen, Hungary

${ }^{c}$ Department of Solid State Physics, Debrecen University, Bem tér 18/b, 4026 Debrecen, Hungary
}

process, since macroscopic observables depend on the morphological and size parameters of the NPs in a rather complex way. ${ }^{13}$ In contrast, the time evolution of observables such as excitonic absorbance can be explicit expressions of reaction rates in the case of one-dimensional particles such as nanowires, provided the total area of growth surfaces is constant and seed formation terminates before the growth period.

Our choice for a model system was the well-established hydrothermal procedure of synthesizing Te nanocrystals in supersaturated solutions of Te atoms or their elemental molecules. These building-block particles (monomers) were produced under mild alkaline conditions via chemical reduction of the sodium tellurite precursor $\left(\mathrm{Na}_{2} \mathrm{TeO}_{3}\right)$ by the reducing agent hydrazine $\left(\mathrm{N}_{2} \mathrm{H}_{4}\right)$ or its protonated form hydrazinium cation $\left(\mathrm{N}_{2} \mathrm{H}_{5}^{+}\right)$, which then turn into inert products in an irreversible process: ${ }^{14,15}$

$$
\begin{array}{cc}
\mathrm{TeO}_{3}{ }^{2-}+\mathrm{N}_{2} \mathrm{H}_{4} \rightarrow \mathrm{Te} \downarrow+\mathrm{N}_{2} \uparrow+2 \mathrm{OH}^{-}+\mathrm{H}_{2} \mathrm{O} \quad E^{\circ}=0.59 \mathrm{eV} \\
\mathrm{TeO}_{3}{ }^{2-}+\mathrm{N}_{2} \mathrm{H}_{5}{ }^{+} \rightarrow \mathrm{Te} \downarrow+\mathrm{N}_{2} \uparrow+\mathrm{OH}^{-}+2 \mathrm{H}_{2} \mathrm{O} \quad E^{\circ}=1.057 \mathrm{eV}
\end{array}
$$

Te nanocrystals preferentially grow into one-dimensional nanowires (NWs) with unlimited length and narrow sizedistribution in diameter under well-controlled physical conditions. ${ }^{16,17}$ Their crystalline form has a trigonal structure (t-Te), which consists of helical chains of covalently bound atoms, and is bundled together by weak secondary interactions. ${ }^{18}$ The unique feature of the longitudinal growth tendency is a consequence of the crystal structure and selective surface stabilization implemented by various additives, which effectively constrain the pathways of crystal growth by preventing 
undesired polymorphism or aggregation as a result of steric stabilization of crystal facets. ${ }^{19}$ As a result, the high-energy surface of t-Te [001] with dangling covalent bonds remains accessible for growth. In our case, PVP was used as a capping and structure-directing agent, ${ }^{20,21}$ which can also act as a suitable crowding agent due to its inertness and high solubility in water. ${ }^{22}$

Similarly to NPs of other semiconducting materials on the scale of quantum-size confinement, excitons of valence to conduction band transitions (VB-CB) appear in tellurium in the UV-Vis spectrum. ${ }^{18}$ These excitons form two absorption peaks at 270-280 nm (VB2-CB1) and 500-650 nm (VB3-CB1), the positions of which shift to longer wavelengths with increasing nanowire dimensions, and can be used to monitor the ripening process. $^{23,24}$

In this work, we report an unprecedented observation of the enhanced growth of Te NWs, which emerged at around the crossover between the semi-dilute and crowding regimes of the PVP concentration. The ripening process of the NWs and a coexisting phase of amorphous Te NPs were monitored by systematically measuring their size-dependent optical properties as a function of time in the UV-Vis spectrum, including excitonic and background parameters. We outline a potential explanation to elucidate the observed rate-enhancement effect on the basis of a simplified association-dissociation reaction scheme and entropic-driven spatial organization of the crowded media.

\section{Experimental section}

\subsection{Chemicals}

All chemicals were used as purchased without any further purification. Hydrazine $\left(\mathrm{N}_{2} \mathrm{H}_{4}, 35 \%\right)$, sodium tellurite $\left(\mathrm{Na}_{2} \mathrm{TeO}_{3}\right)$ and PVP powder $\left(M_{\mathrm{w}} \approx 10000\right)$ were purchased from Alfa-Aesar and Sigma-Aldrich companies. The $\mathrm{pH}$ of the PVP solutions in the present study ranged between 4.5 and 7. Water from a Milli-Q ultrapure system was used for the preparation of the solutions.

\subsection{Synthesis of Te nanowires}

Te NWs were synthesized via two different procedures (Fig. 1). The synthesis solutions for the base procedure (procedure A) were first prepared at $60{ }^{\circ} \mathrm{C}$ by mixing $1 \mathrm{ml}$ of $1.5 \mathrm{M} \mathrm{N}_{2} \mathrm{H}_{4}$ solution as a reducing agent and $1 \mathrm{ml}$ of PVP solution with concentrations systematically varied in every synthesis batch. The synthesis was carried out in a $10 \mathrm{~mm}$ path-length quartz optical cuvette connected to a regulated heat reservoir and placed in a spectrophotometer. The volume of the reaction batch was $3 \mathrm{ml}$. Adding $1 \mathrm{ml}$ of $45 \mathrm{mM} \mathrm{Na}_{2} \mathrm{TeO}_{3}$ solution to the prepared mixture took place at $80{ }^{\circ} \mathrm{C}$, and then the cuvette was pressure closed with Teflon sealing and heated gradually to a synthesis temperature of $105{ }^{\circ} \mathrm{C}$ at a heating speed of $5{ }^{\circ} \mathrm{C} \mathrm{min}{ }^{-1}$. The batch solution contained $15 \mathrm{mM} \mathrm{Na}_{2} \mathrm{TeO}_{3}$ and $500 \mathrm{mM}$ $\mathrm{N}_{2} \mathrm{H}_{4}$, as well as PVP with concentrations varied in the range of $c_{\mathrm{PVP}}=0.0167-132 \mathrm{~g} \mathrm{l}^{-1}$. The initial $\mathrm{pH}$ values of the respective batch solutions ranged from 10.6 to 10.0 .

In a seeded-growth procedure (procedure B) synthesis batches were prepared by mixing $1 \mathrm{ml}$ of identical aliquots

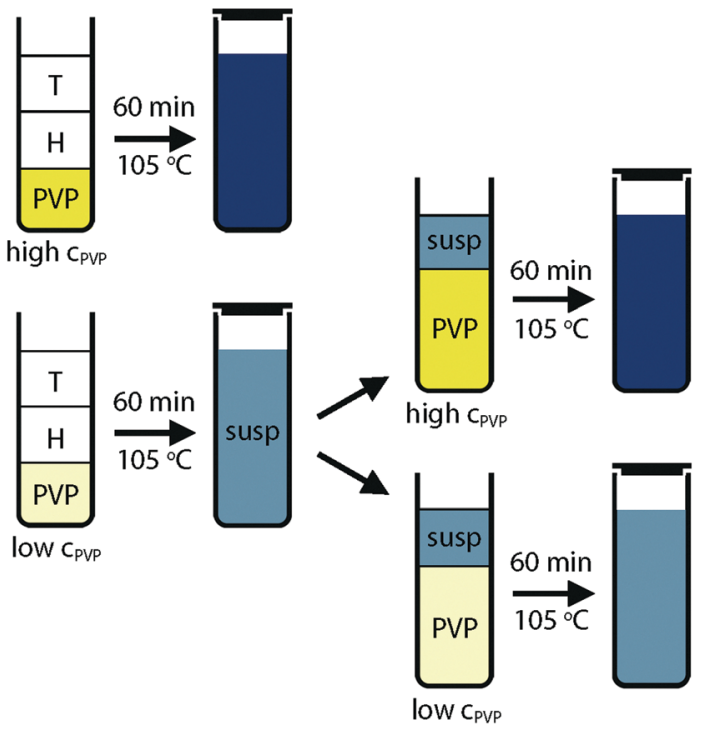

procedure A

procedure B

Fig. 1 Schematic illustration for the synthesis of Te nanowires starting from either the initial reactants of $\mathrm{Na}_{2} \mathrm{TeO}_{3}(\mathrm{~T})$ and $\mathrm{N}_{2} \mathrm{H}_{4}(\mathrm{H})$ in procedure $A$ or a mature nanowire suspension (susp) in procedure $\mathrm{B}$. The reactants are shown in the order of addition of their solutions.

from an NW suspension previously synthesized in procedure A and $2 \mathrm{ml}$ of PVP solutions with varying concentrations. The NW suspension was prepared in a $100 \mathrm{ml}$ flask containing $10 \mathrm{ml}$ of each stock solution using $c_{\mathrm{PVP}}=1.67 \mathrm{~g} \mathrm{l}^{-1}$, under vigorous stirring for $60 \mathrm{~min}$ at $105{ }^{\circ} \mathrm{C}$. The batch concentration of PVP was set in five steps in the range of $c_{\mathrm{PVP}}=3.89-166 \mathrm{~g} \mathrm{l}^{-1}$. The NW suspension and the PVP stock solution were mixed at room temperature, and the $\mathrm{pH}$ was set to a common value of 9.1 by adding $10-50 \mu \mathrm{l}$ of $0.4 \mathrm{M} \mathrm{HCl}$ solution. The prepared mixture was filled into the cuvette, and heated up to $105{ }^{\circ} \mathrm{C}$ following the protocol of procedure A measurements.

In the reaction-order measurements based on the protocol of procedure $\mathrm{B}$, the volume of the injected suspension was systematically varied from 0.25 to $2 \mathrm{ml}$, keeping the PVP concentration fixed at $33.8 \mathrm{~g} \mathrm{l}^{-1}$.

\subsection{Measurements}

The optical measurements were performed with a SPECORD S600 UV-Vis spectrophotometer from Analytik Jena. The absorbance spectra were recorded in 10 second steps for $60 \mathrm{~min}$ starting when the temperature of the batch solution reached $85{ }^{\circ} \mathrm{C}$ during the heatup period. The spectra covered the 180-1000 nm wavelength region. Reference spectra were measured using Milli-Q water before every measurement. Spectra of the reactants and the PVP concentration series were also recorded to subtract their contribution from spectra of the synthesized suspension. A typical measurement taken at subsequent stages of the synthesis is illustrated in Fig. 2a.

\subsection{Characterization}

The morphology of the synthesized NWs and NPs was examined with a JEOL JEM-2000FX transmission electron microscope 

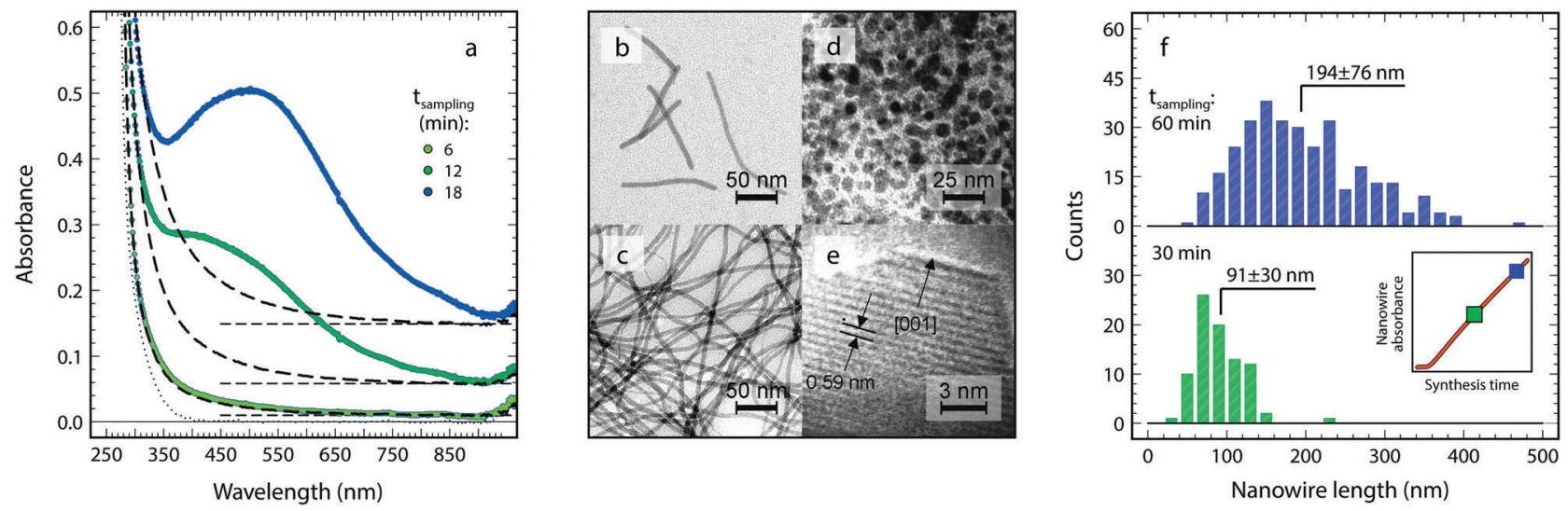

Fig. 2 (a) Absorbance spectra measured at subsequent stages of NW synthesis and their decomposition to excitonic and background contributions. The spectra demonstrate the protrusion and the redshift of the excitonic peak, which superimpose on the Rayleigh-scattering spectrum of amorphous Te NPs following a simple $\lambda^{-4}$ function (dashed curves). The dotted curve corresponds to the contribution of the initial reactants and the PVP. The dashed lines show the baseline of the Rayleigh-scattering background, which is identified as a further background component (see the text). (b and c) TEM images recorded at early and well-developed stages of the NW synthesis. (d) TEM image of the spherical-like, amorphous Te NPs. (e) High-resolution TEM image of the end-section of an NW to provide proper characterization of the microstructure and growth direction of the NWs. (f) Length distributions of the NWs from the analysis of TEM images. The samples were taken at different synthesis times from the same synthesis batch with $c_{\mathrm{PVP}}=$ $0.167 \mathrm{~g} \mathrm{l}^{-1}$. The comparison illustrates the direct relationship between the longitudinal growth of the NWs and the increase in the averaged absorbance shown in the inset figure.

(TEM) as shown in Fig. 2b-e. The samples were prepared by drying under ambient conditions without any dilution or mechanical processing to preserve their as-synthesized structure. Fig. $2 \mathrm{~b}$ and $\mathrm{c}$ show typical bunches of the NWs at early and advanced stages of the synthesis. To provide proper characterization of the microstructure and growth direction of the NWs, a high-resolution TEM image was also recorded as shown in Fig. 2e.

\subsection{Data reduction}

The absorbance contributions of the initial reactants and the dissolved PVP were first subtracted from the measured spectra. Since the absorbances of the $\mathrm{N}_{2} \mathrm{H}_{4}$ and $\mathrm{Na}_{2} \mathrm{TeO}_{3}$ solutions have a steep rise in the mid-UV region, the NW absorbance cannot be evaluated below the wavelength of $250 \mathrm{~nm}$. The corrected spectra were then decomposed to a smooth background and to an excess yield stemming from the exciton of t-Te. The background was further decomposed to components differing in their wavelength dependence, one exhibiting a $\lambda^{-4}$ function related to Rayleigh-scattering and a constant (wavelength-independent) background. ${ }^{25}$ The sum of these functions was fitted on the spectra in wavelength regions where the excitonic yield is negligible. These regions are typically located between 280 and $350 \mathrm{~nm}$ (NUV) and 900 and $1000 \mathrm{~nm}$ (NIR). The absorbances of all the spectral components (excitonic peak, Rayleigh-scattering and constant backgrounds) were determined for every time step, and averaged over the $330-900 \mathrm{~nm}$ region. Similarly, the centre of the excitonic peak was also deduced as the absorbanceweighted average of the excitonic distribution over the same wavelength region. The sample-to-sample variation of integrated absorbance values originated from both dosing uncertainties and systematic errors of the fitting procedure, which was estimated from repeated measurements to be less than $15 \%$ and $40 \%$ for the excitonic and Rayleigh-scattering components, respectively.
The particle-size distributions were generated by unbiased sampling from the TEM images. The centroid and width parameters of the distributions were found rather insensitive to the sample-to-sample variation due to the relatively large standard deviation which is the consequence of the limited number of particle specimens visually observed.

\section{Results and discussion}

The synthesis of nanosized t-Te crystals based on procedure A was observed as the growing excitonic absorbance of the VB3-CB1 transition, which is located in the visible spectrum as shown in Fig. 2a. At different synthesis times, the measured absorbance demonstrates the protrusion of the excitonic structure above a smooth background. The exciton of the VB2-CB1 transition, usually measured in the UV spectrum at 270-280 nm, ${ }^{23,26}$ could not be resolved due to the steep increase in the absorption of tellurite and PVP solutions.

The absorbance-weighted centre of the visible excitonic peak shifted from approximately $500 \mathrm{~nm}$ to $600-650 \mathrm{~nm}$ during the 60 min course of the synthesis. The analysis of TEM images, partly shown in Fig. 2b-e, and related energy-dispersive X-ray (EDX) data supported the presence of 1-dimensional Te nanostructures in the forms of nanorods and nanowires. The images also revealed a coexisting phase of spherical-like particles with diameters of 5-15 nm as shown in Fig. 2d, which were identified as amorphous Te NPs similarly to previous experiments. ${ }^{15,23}$ The continuous development of nanorods to nanowires and their longitudinal growth were observed in TEM images (Fig. 2b and c) and in the corresponding length distributions (Fig. 2f). Moreover, the diameter of the NWs remained constant along the growth axis due to the stabilizing effect of PVP, and its 
distribution typically peaked at $6 \pm 1 \mathrm{~nm}$. Based on these observations, the wavelength-averaged absorbance of the excitonic peak is assumed to be a linear function of the cumulated NW length, and its time-derivative can give direct information on growth rates.

During the induction period preceding the visible formation of the crystalline phase, a structureless spectrum appears as a monotonously decreasing function of wavelength as seen in Fig. 2a. This spectrum is characteristic of optical extinction caused by scattering on particles with sizes smaller than the wavelength of the incident light. Evaluating the TEM images, as exemplified in Fig. 2d, spherical particles are sufficiently small to use Rayleigh approximation on their spectra (Rayleighscattering absorbance), which gives a satisfactory fit with a $\lambda^{-4}$ function to the background under the emerging excitonic structure. ${ }^{25}$ The excitonic peak then gradually becomes visible due to the developing crystalline phase, and the excess yield above the Rayleigh-scattering absorbance is referred to as NW absorbance. It is apparent that NW absorbance can be further decomposed to the localized structure of the excitonic peak and a background nearly independent of the wavelength. The latter component was also fitted in every time step and found to be linearly proportional to the averaged absorbance of the excitonic peak in the growth period. Based on self-consistent calculations on t-Te crystals, the imaginary part of the transversal and longitudinal components of the frequency-dependent permittivity considerably differ for the VB3-CB1 transitions. ${ }^{27}$ While the former yields a relatively sharp peak around $2 \mathrm{eV}$ corresponding to the observed excitonic structure, the latter results in a broader structure over the entire visible spectrum extending to the near-infrared. Rayleigh scattering on the NWs as a non-excitonic extinction process may also be present in the background, although its contribution to the integrated yield is potentially less dominant partly due to the isotropic distribution of the optical axis of the NWs and the polarization of the incident light, as well as to the reduced dielectric contrast at the diffuse capping layer on the crystal-polymer interface.

The relevant quantities of the spectral components (the centre of the excitonic peak, and nanowire and Rayleigh-scattering absorbance) are presented in Fig. 3 as a function of synthesis time and parametrised by the systematically varied PVP concentration. Three stages of the ripening process can be recognized in the evolution curves. The first section lasting for 5-10 min, including the heat-up period when the temperature was increased to $105{ }^{\circ} \mathrm{C}$, is the induction period, which is characterised only by the formation of amorphous NPs. The second section in the interval of 5-15 min is the seeding period, when the excitonic peak turns up and rapidly shifts to a longer wavelength. This behaviour was pointed out in previous experiments, which revealed a correlation between the nanorod length and the
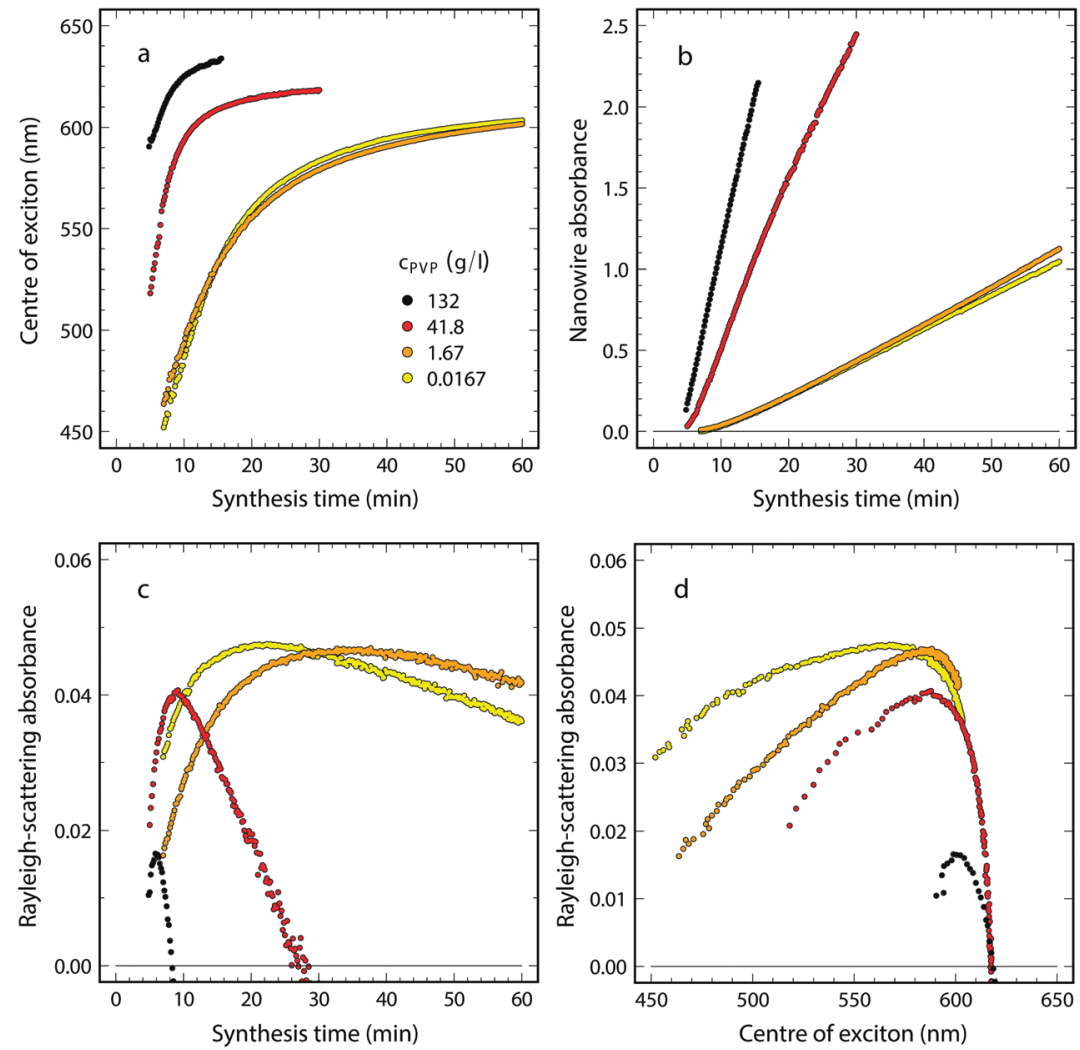

Fig. 3 Time evolution of relevant parameters of absorbance components for PVP concentrations ranging from dilute to crowding regimes: (a) absorbance-weighted centre of the VB3-CB1 excitonic peak; (b) averaged absorbance of Te NWs after subtracting the contribution of Rayleigh scattering; (c) averaged absorbance of Rayleigh-scattering; and (d) correlation plot of the Rayleigh-scattering absorbance and the centre of the excitonic peak. 
centre of the excitonic peak; ${ }^{23}$ however, its radial dependence is presumably irrelevant during the synthesis, since the nanorod diameter does not change after crystalline seeds are formed. In parallel, the Rayleigh-scattering absorbance steeply increases before it reaches a maximum value. After the length of the growing nanorods exceeds the size of the Bohr-exciton of bulk t-Te, the length dependence vanishes and the centre of the exciton converges to $610-630 \mathrm{~nm}$. At this stage of evolution, the NW absorbance realizes a linear time dependence, while the Rayleigh-scattering absorbance turns to a declining section. Taking into account the typical power-law dependence of the Rayleigh-scattering cross section on the particle diameter $\left(\sigma_{\mathrm{R}} \propto d^{6}\right)$, the declining behaviour may be attributed to the dissolution of the NPs. ${ }^{25}$

The most striking and common feature of all evolution data is the monotonously accelerated ripening of both the NW and Rayleigh-scattering absorbances with increasing PVP concentration (Fig. 3). The drastic enhancement of growth rates took place only above $\approx 20 \mathrm{~g} \mathrm{l}^{-1}$ typically falling in the crossover region between semi-dilute and crowding concentrations, while at lower concentrations no significant effect of PVP was found. It is an obvious question as to whether the faster processes connected with higher monomer consumption rates are caused by higher concentrations of crystalline seeds or by higher longitudinal growth rates of the NWs. Upon comparing the size parameters of the NWs synthesized at semi-dilute and crowding concentrations of PVP, as shown in Fig. 4a and b, the substantial difference in length suggests that crowding promotes longitudinal growth, while significant change in the average radii was not found. Consequently, crystal-seed concentrations might have only a minor or negligible role in the observed effect.

The size distribution and concentration of premature particles sensitively depend on the uncertainties of the initial parameters due to the stochastic nature of nucleation. Furthermore, as the $\mathrm{pH}$ of the initial batch mixtures was measured in the range of 10.0-10.6 depending on the amount of PVP applied, it may affect the relative concentration of hydrazine and its protonated form (hydrazinium cation), which potentially results in increased growth rates considering the difference in the electromotive force of the respective reduction processes. To eliminate the role of these effects and uncertainties, we extended procedure A to a seeded-growth synthesis (procedure B) with controlled concentration parameters and $\mathrm{pH}$ adjusted to $9.10 \pm 0.05$ by the addition of $0.4 \mathrm{M} \mathrm{HCl}$ solution. NW suspension prepared for 60 min under semi-dilute conditions was injected in identical amounts to PVP solutions ranging from semi-dilute to crowding concentrations, and ripened for another $60 \mathrm{~min}$ period at $105^{\circ}$. The NWs and their length distributions at the end of the procedure-B synthesis are illustrated in Fig. 5 for semi-dilute and crowding PVP concentrations. During the course of the synthesis, the $\mathrm{pH}$ decreased by an amount of 0.1-0.2 with respect to the adjusted value. Considering that the concentrations of NWs and NPs, as well as of any remaining reactant, are equal in each synthesis batch, the procedure is capable of verifying that crowding affects the crystal-growth dynamics instead of the nuclei formation. After a short heat-up period,
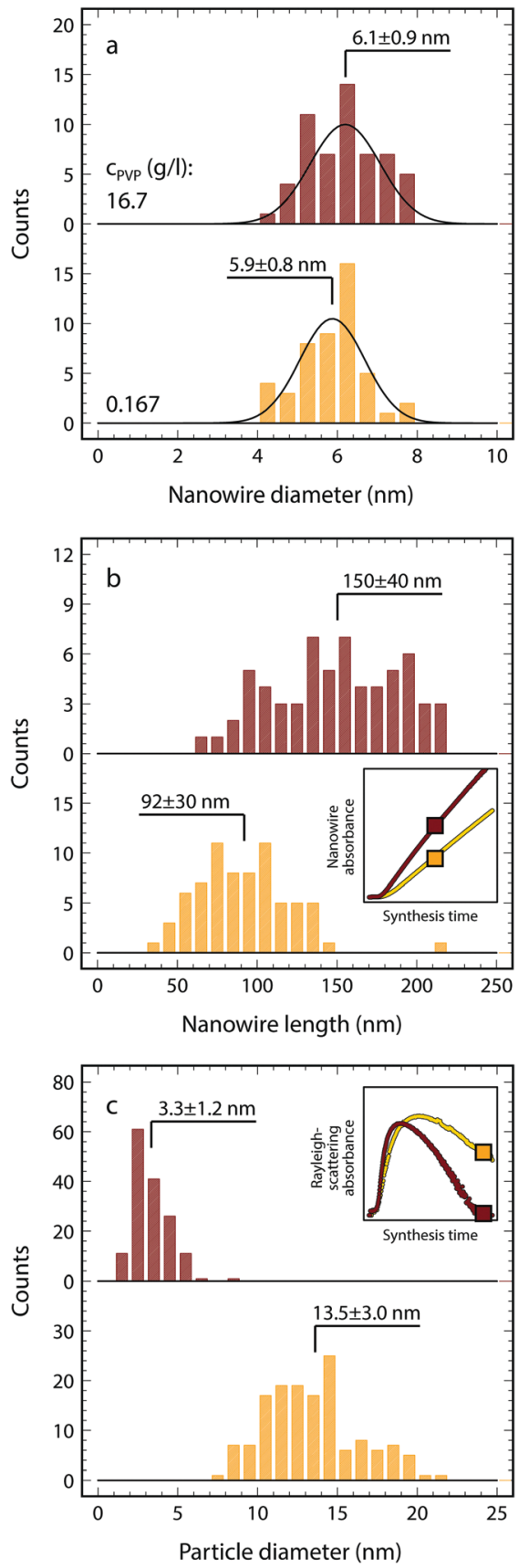

Fig. 4 Comparison plots of particle-size distributions representing synthesis batches with $C_{P V P}=0.167$ and $16.7 \mathrm{~g} \mathrm{l}^{-1}$ given in the bottom and top parts of each panel, respectively. The histograms were deduced from the evaluation of TEM images: (a) diameter distribution of NWs approximated with normal distributions; (b) length distributions of NWs, where the inset figure indicates the sampling time of $30 \mathrm{~min}$ in the evolution curves of NW absorbance; and (c) diameter distribution of amorphous NPs, where the inset figure indicates the sampling time of $60 \mathrm{~min}$ in the evolution curves of Rayleigh-scattering absorbance.

the time evolution of the NW absorbance in Fig. 6a quickly turns to a monotonously increasing section, the slope of which demonstrates a significant enhancement with increasing PVP concentration similarly to the systematics measured in procedure A. The negative deviation from a linear approximation is probably 

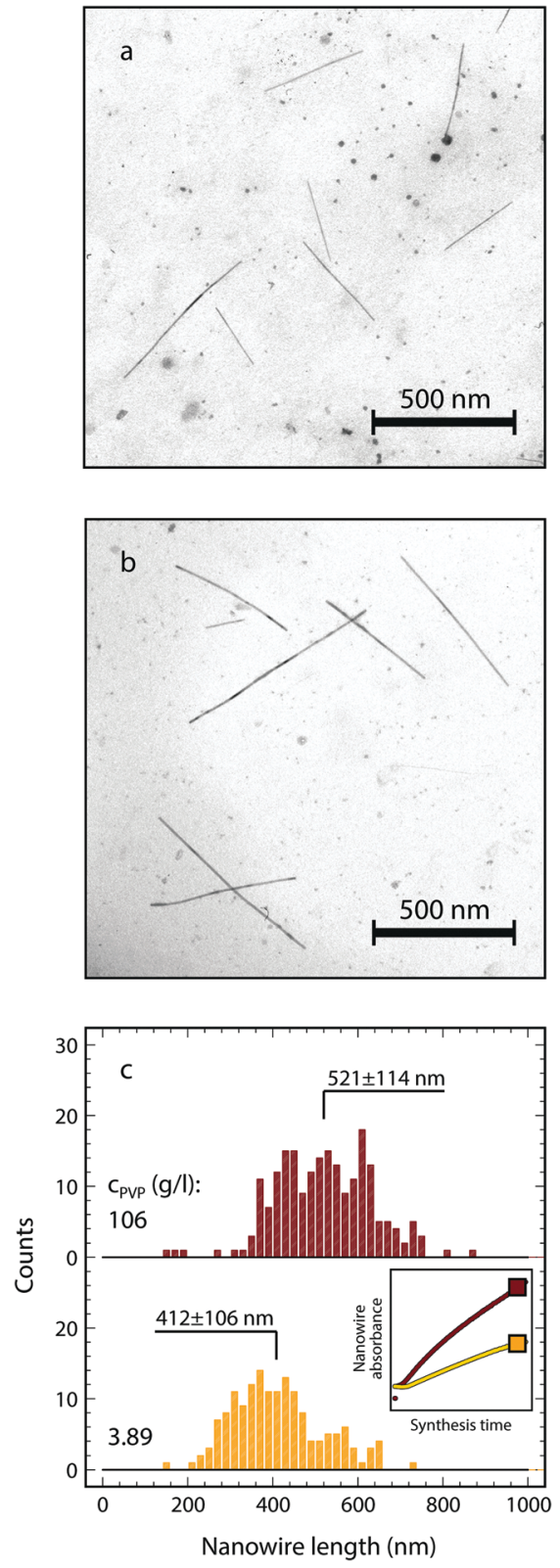

Fig. 5 TEM images of Te NWs after the 60 min course of procedure-B synthesis for PVP concentrations: (a) $3.89 \mathrm{~g} \mathrm{l}^{-1}$ and (b) $106 \mathrm{~g} \mathrm{l}^{-1}$. (c) Nanowire-length distributions determined from the TEM images exemplified in panels $a$ and $b$. The weighted means and standard deviations of the distributions are also given.

caused by the gradual exhaustion of the monomers. The declining section of the Rayleigh-scattering absorbance evolution was also approximated with a linear model for procedure-A data, while the slopes deduced from the procedure-B data could not be determined with acceptable confidence, because the mean size of the amorphous NPs fell below the threshold of optical sensitivity. The time derivative of the nanowire absorbance is summarized in Fig. $6 \mathrm{~b}$ as a function of PVP concentration at a fixed $\mathrm{pH}$. To quantify the sample-to-sample reproducibility of growth data, the statistical analysis of five independent series of procedure-B synthesis was performed, and errors comprising both systematic and statistical uncertainties of data reduction were determined and are shown in Fig. $6 \mathrm{~b}$.

The amount of the injected suspension in procedure B was also varied at fixed PVP concentrations to determine the reaction-rate exponent for the crystal growth, which was found to be $2.09 \pm 0.05$ by fitting the slope parameter in the linear-growth section. This reveals that the overall process can be viewed as a purely binary reaction between the NWs and the monomers (Fig. 6c).

For a deeper understanding of the molecular mechanisms behind the observed rate-enhancement effect of MMC, a plausible reaction scheme is outlined based on both thermodynamic and structural considerations of the associationdissociation dynamics (Fig. 7).

Initially, the decomposition of precursors by chemical reduction leads to the instantaneous supersaturation of zerovalent species, functioning as monomers for spontaneously forming nuclei. The formation of such new-phase particles then suddenly pulls down the concentration of the monomers, which then terminates the nucleation; the solution becomes undersaturated, and eventually makes the particles subcritical. ${ }^{28,29}$ In accordance with the Ostwald-step rule, nuclei with a preferentially metastable, often disordered state are formed, which are structurally closest to their parent state. ${ }^{30}$ Later, particles may acquire a partial crystalline structure as a transitional state (e.g. crystal facets or edges) and form seeds for subsequent crystal growth, which possibly remain supercritical due to their lower solubility and higher surface energy with respect to the amorphous NPs. The crystal seeds then undergo a process, in which the dissolution of the disordered domain and the epitaxial growth of the crystal facets take place in parallel. The process can be viewed as a special case of Ostwald ripening, where the size-controlled process described by the Gibbs-Thomson equation is replaced by structural control, sometimes referred to as intraparticle growth. ${ }^{28}$

The ascending section of the Rayleigh-scattering absorbance as shown in Fig. 3c in procedure A is considered as a sign of Ostwald ripening of the amorphous population taking into account that only the fraction of the largest particles is visible in the spectra. After the first crystalline seeds start growing, the amorphous population undergoes a dissolution process, the rate of which correlates with the rate of NW growth in the linear section at all PVP concentrations applied. This behaviour of evolution is demonstrated in Fig. 3d, where points of the Rayleigh-scattering absorbance follow very similar tracks and converge to practically the same position of the excitonic centre in the linear-growth section.

Evaluating the reaction-order measurement on procedure $\mathrm{B}$, a partial order of approximately one can be assigned to the concentration of the monomers, which is the only dynamic quantity that determines the NW growth. When realizing a balance between the source and consumption rates of monomers during the linear-growth period, the monomer saturation takes a constant, non-vanishing value. In this steady state, the monomer-association process is assumed to be the ratelimiting step, on which the influence of the crowded environment must be examined on the basis of the dynamic control over the geometrically constrained space. 

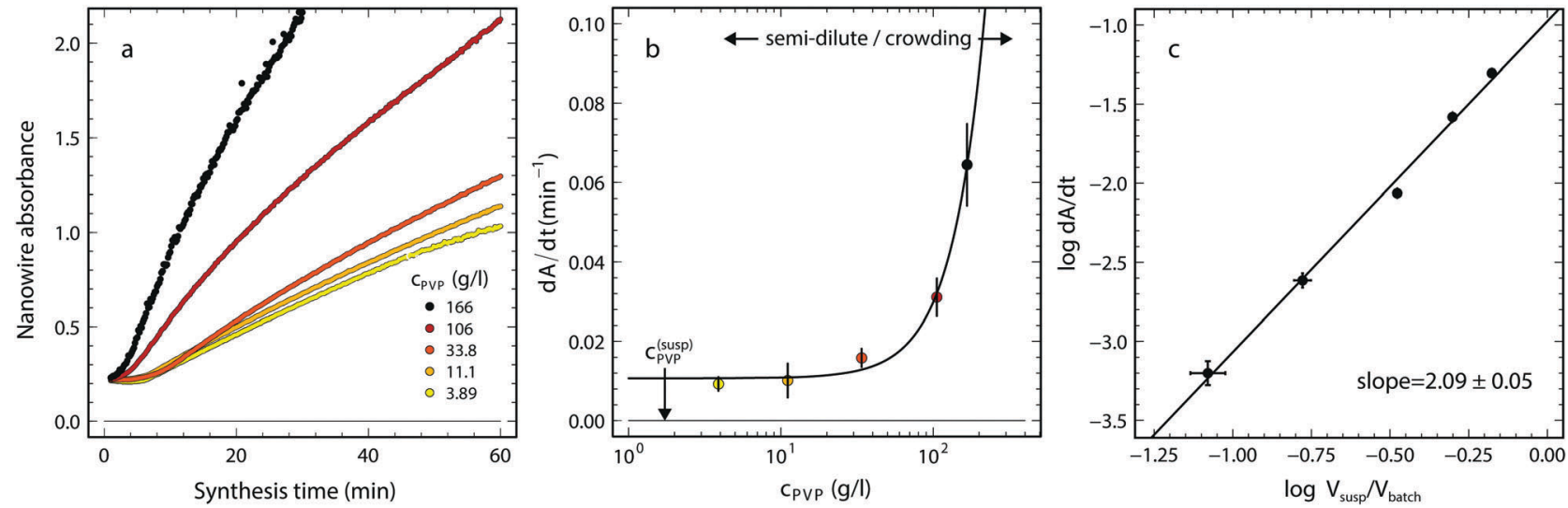

Fig. 6 (a) Time evolution of the averaged absorbance of the synthesis batch in the seeded-growth synthesis of procedure $\mathrm{B}$. The $\mathrm{pH}$ of each synthesis batch was adjusted to $9.10 \pm 0.05$ by the addition of $0.4 \mathrm{M} \mathrm{HCl}$ solution. (b) Time derivative of the nanowire absorbance from procedure-B data as a function of $C_{\text {PVP }}$ at the adjusted $\mathrm{pH}$. The error bars represent the systematic and statistic uncertainties determined from five independent series of measurements. The solid line shows a second-order polynomial fit to the data points. (c) Reaction-rate exponent determined for the synthesis of Te NWs based on the protocol of procedure B with different amounts of injected NW suspensions $\left(V_{\text {susp }}\right)$ at fixed $C_{\text {PVP }}=33.8 \mathrm{gl}^{-1}$. The logarithmic values were fitted with a linear model.

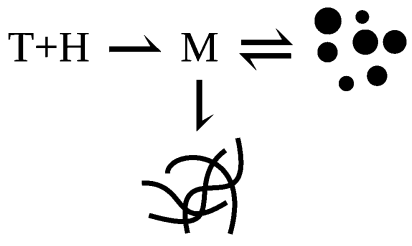

Fig. 7 Principal reaction scheme of the association-dissociation dynamics between the building-block monomers $(\mathrm{M})$ and the two populations of tellurium nanowires and amorphous particles. The primary reactants are sodium tellurite $(\mathrm{T})$ and hydrazine $(\mathrm{H})$. Monomeric currents of the precursor reduction and nanowire growth are assumed to be unidirectional.

Note that crowded media of inert obstacles naturally lead to an obstructed diffusion, ${ }^{31}$ which suppresses the rates of kinematically controlled processes. The obstructed diffusion may have a selective effect on reaction pathways with different reaction orders. In the simplest case, the associative processes are disfavoured against the dissociative ones; consequently, the effective solubility of the particles must increase. Since the consumption rate of the monomers governed by the associativerate constant of NW growth is then also suppressed, the monomer concentration increases toward the solubility region of the amorphous population. Potentially, this would lead to the Ostwald ripening of the NPs; however, this scenario would confront with the observations of shrinking NP size-distribution in Fig. 4c. Although this effect may still be present in the limit of kinematic control, it might have only a minor role in the overall growth process.

In thermodynamic control, the role of kinematic obstruction must principally be different due to the structural organization of the media, which might elucidate the accelerated NW growth. In fact, the associative reactions are sequential processes of distant transport and local random walk of the reactants. The former process is quantified by the so-called first-passage time; in our case, this is the time needed for a mobile reactant to first encounter the NW end. However, this process is slowed down in a crowded environment, which can be overbalanced by increased recollision rates. ${ }^{32,33}$ This case can be realized if reactants are temporally trapped in compartments that force them to explore the local environment on a time scale much longer than defined by the recollision frequency.

The formation of compartments is ascertained by the entanglement of polymeric chains at the surface of the newphase particles due to the partially overlapping excluded volumes of the crowder and capping molecules. This interaction is of entropic nature and creates a depletion zone expelling the crowder molecules. In contrast, the depletion zones trap the monomers by maintaining a dynamic balance between their diffusion along the concentration gradient toward the bulk solution and the driving force by the entropic gain of their localization in the depletion zone.

The caging of monomers inside the compartments constrains their random distribution in the phase space; hence, their configurational entropy is reduced with respect to the unconstrained case of dilute solutions. The chemical potential of the monomers in the solution phase should also increase accordingly, ${ }^{34}$ which leads to higher transition probabilities through the activation barrier; therefore, less free energy is required to reach the transition states. The outlined approach can reasonably be phrased as structural catalysis induced by the compartmentalized space of MMC.

\section{Conclusions}

We have investigated the role of MMC in the wet-chemical synthesis of Te nanowires using PVP as both a capping and crowding agent in a wide range of concentrations. The ripening process of the nanowires and the coexisting phase of amorphous Te nanoparticles were monitored by systematically measuring their size-dependent optical properties as a function of time. Shortly after the induction period, the nanowire growth entered a steady state observed as a linear increase of the 
excitonic absorbance, which was significantly enhanced with increasing PVP concentration above the crossover between semidilute and crowding regimes. In parallel, the evolution of amorphous nanoparticles characterized by their simultaneous dissolution was also accelerated with increasing PVP concentration.

We outlined a possible explanation for the crowdinginduced enhancement of nanowire growth on the basis of the association-dissociation reaction scheme and the non-specific structural organization of the crowded media. Associative processes may be governed by the complex kinematics due to space compartmentalization localized to the active surfaces of the new-phase particles, ultimately leading to a catalysed process in the limit of thermodynamic control.

Motivated by the presented methodology, the effect of MMC on the synthesis of tellurium nanowires as a specific model system for investigating phase transitions and phase behaviour in a more general sense is proposed to be tested by further experimental and simulation studies, which potentially give rise to a more fundamental understanding of crowded systems.

\section{Acknowledgements}

The work was supported by the GINOP-2.3.2-15-2016-00041 project, which is co-financed by the European Union and the European Regional Development Fund.

\section{References}

1 A. P. Minton, Biopolymers, 1981, 20, 2093-2120.

2 G. B. Ralston, J. Chem. Educ., 1990, 67, 857-860.

3 R. J. Ellis, Trends Biochem. Sci., 2001, 26, 597-604.

4 R. J. Ellis and A. P. Minton, Nature, 2003, 425, 27-28.

5 A. H. Elcock, Curr. Opin. Struct. Biol., 2010, 20, 196-206.

6 A. Rao and H. Cölfen, J. Struct. Biol., 2016, 196, 232-243.

7 T. L. Doane and C. Burda, Chem. Soc. Rev., 2012, 41, 2885-2911.

8 G. Bao, S. Mitragotri and S. Tong, Annu. Rev. Biomed. Eng., 2013, 15, 253-282.

9 H. F. Krug and P. Wick, Angew. Chem., Int. Ed., 2011, 50, 1260-1278.

10 R. H. Müller, S. Gohla and C. M. Keck, Eur. J. Pharm. Biopharm., 2011, 78, 1-9.

11 H. N. W. Lekkerkerker and R. Tuinier, Lect. Notes Phys., 2011, 833, 57-108.
12 A. P. Minton, Biopolymers, 2013, 99, 239-244.

13 Y. Xia, P. Yang, Y. Sun, Y. Wu, B. Mayers, B. Gates, Y. Yin, F. Kim and H. Yan, Adv. Mater., 2003, 15, 353-389.

14 Z. Liu, S. Li, Y. Yang, Z. Hu, S. Peng, J. Liang and Y. Qian, New J. Chem., 2003, 27, 1748-1752.

15 H.-S. Qian, S.-H. Yu, J.-Y. Gong, L.-B. Luo and L.-f. Fei, Langmuir, 2006, 22, 3830-3835.

16 H. Zhu, H. Zhang, J. Liang, G. Rao, J. Li, G. Liu, Z. Du, H. Fan and J. Luo, J. Phys. Chem. C, 2011, 115, 6375-6380.

17 H. Park, W. Son, S. Hwan Lee, S. Kim, J. Joon Lee, W. Cho, H. Hee Choi and J. Hyun Kim, CrystEngComm, 2015, 17, 1092-1097.

18 P. Ghosh, M. U. Kahaly and U. V. Waghmare, Phys. Rev. B: Condens. Matter Mater. Phys., 2007, 75, 245437.

19 B. A. Rozenberg and R. Tenne, Prog. Polym. Sci., 2008, 33, 40-112.

20 F. Haaf, A. Sanner and F. Straub, Polym. J., 1985, 17, 143-152.

21 W. A. Al-Saidi, H. Feng and K. A. Fichthorn, Nano Lett., 2012, 12, 997-1001.

22 A. C. Miklos, C. Li, N. G. Sharaf and G. J. Pielak, Biochemistry, 2010, 49, 6984-6991.

23 Z.-H. Lin, Z. Yang and H.-T. Chang, Cryst. Growth Des., 2008, 8, 351-357.

24 J. Jasieniak, M. Califano and S. E. Watkins, ACS Nano, 2011, 5, 5888-5902.

25 C. F. Bohren and D. R. Huffman, Absorption and Scattering of Light by Small Particles, Wiley-VCH Verlag, 1998.

26 Y. Li, W.-Y. Zhao, X. Mu, X. Liu, D.-Q. He, W.-T. Zhu and Q.-J. Zhang, J. Electron. Mater., 2016, 45, 1661-1668.

27 H. M. Isomäki and J. von Boehm, Phys. Scr., 1982, 25, 801-803.

28 N. T. K. Thanh, N. Maclean and S. Mahiddine, Chem. Rev., 2014, 114, 7610-7630.

29 E. R. Leite and C. Ribeiro, Crystallization and Growth of Colloidal Nanocrystals, SpringerBriefs in Materials, 2012.

30 R. A. Van Santen, J. Phys. Chem., 1984, 88, 5768-5769.

31 J. A. Dix and A. S. Verkman, Annu. Rev. Biophys., 2008, 37, 247-263.

32 M. C. Buján-Nuñez and M. A. Lopez-Quintela, J. Chem. Phys., 2004, 121, 886-889.

33 O. Bénichou, C. Chevalier, J. Klafter, B. Meyer and R. Voituriez, Nat. Chem., 2010, 2, 472-477.

34 D. Marn, M. Martn and B. Sabater, BioSystems, 2009, 98, 31-36. 